\title{
MIRADAS A AMÉRICA LATINA
}

\author{
Rafael Rojas \\ El Colegio de México
}

Ta revista Historia Mexicana fue fundada en 1951 por Daniel Cosío Villegas y un grupo selecto de historiadores mexicanos con el objetivo de propiciar un foro de debate profesional sobre la historia de México a mediados del siglo xx. Uno de los miembros del primer núcleo editor, Wigberto Jiménez Moreno, lamentaba en 1952 la ausencia de una revista especializada en la historiografía nacional durante la primera mitad de la centuria. ${ }^{1}$ Más allá de que lo "profesional" de aquella historiografía estuviera muy lejos de las normas académicas contemporáneas, la prioridad de la publicación fue trazada a favor del avance del conocimiento histórico nacional.

Desde la revolución mexicana, sin embargo, la posición del país en el sistema internacional y, especialmente, dentro de la región latinoamericana, había adquirido una relevancia notable. El Colegio de México, lo mismo que el Fondo de Cultura Económica, fundados ambos por Daniel Cosío Villegas, tenían una evidente proyección continental, que se reforzó durante la

1 Jiménez Moreno, “50 años de historia mexicana” (3), 1952, pp. 449-455, esp. pp. 451-452. 
Guerra Fría. Aunque concebida para actualizar y difundir la producción historiográfica nacional, Historia Mexicana sostuvo, desde sus inicios, una mirada hacia el acontecer intelectual latinoamericano. En las páginas que siguen se propone, a grandes rasgos, una reconstrucción de la presencia de América Latina en las páginas de la revista.

\section{LA QUERELLA HISTORIOGRÁFICA}

Una de las primeras menciones de América Latina en Historia Mexicana apareció en la reseña del libro de Harry Bernstein, Modern and Contemporary Latin America (1952), que escribió María de Carmen Velázquez para el sexto número. Esta historiadora cuestionaba la selección de países tratados en el libro del profesor de Brooklyn College, quien luego escribiría un conocido ensayo sobre Matías Romero. Bernstein utilizaba en su libro el concepto de América Latina, pero sólo se ocupaba de México, Argentina, Brasil, Chile y Colombia. Según Velázquez no era esa la única limitación del libro: también reprochaba el peso del determinismo económico, que traducía como "materialismo", y la ausencia de una visión de conjunto de la que llamaba "América española y portuguesa". ${ }^{2}$

Velázquez formó parte de la tercera promoción del Centro de Estudios Históricos (CEH) y se especializó en el impacto de las guerras borbónicas en la Nueva España, a fines del siglo Xviır. Su idea de una América Latina total, marcada desde los siglos coloniales por las instituciones y leyes de las monarquías ibéricas, estaba muy arraigada en la visión de su maestro Silvio Zavala. La presencia de estudiantes latinoamericanos desde las primeras generaciones del CEH, en los años cuarenta (las puertorriqueñas Monelisa Lina Pérez Marchand e Isabel Gutiérrez del Arroyo, el guatemalteco Ernesto Chinchilla, el venezolano Eduardo

2 Velázquez, "De nuevo la América Latina” (6), 1952, pp. 277-278. 
Arcila Farías, el colombiano Germán Posada, la costarricense Ligia Cavallini y los cubanos Julio Le Riverend, Manuel Moreno Fraginals y Carlos Funtanellas), contribuyó a completar el mapa de América Latina desde los primeros años de la revista. ${ }^{3}$

Era aquel un latinoamericanismo que, a diferencia del hispanismo de la primera década del Centro de Estudios Históricos, estaba ligado a las redes interamericanas y las fuertes relaciones culturales con el campo intelectual y el mundo universitario de Estados Unidos, que caracterizaron a la vida académica mexicana en el arranque de la Guerra Fría. La importancia de las relaciones con Estados Unidos en la proyección misma del latinoamericanismo se observa en Historia Mexicana desde el breve ensayo de Charles E. Roman en que refutaba la etimología peyorativa que se daba a la palabra "gringo", supuestamente surgida en tiempos de la guerra de 1847, por algún uso del verso "Green grow the rashes" de la famosa canción de Robert Burns. La nutrida nómina de historiadores estadounidense que colaboraron en la revista desde sus orígenes (Stanley Ross, Jack. D. L. Holmes, Hugh H. Hamill, Frank A. Knapp, Robert A. Potash, Charles Hale, Herbert S. Klein...) da cuenta de esa conexión. ${ }^{4}$

Al cumplirse la primera década de Historia Mexicana, su director fundador Daniel Cosío Villegas escribió un breve texto titulado "Diez años de camino", que antecedió el primer Índice de la publicación, compilado por el peruano Luis Muro, también discípulo de Zavala. ${ }^{5}$ Allí Cosío Villegas señalaba que el surgimiento de la revista había sido posible porque la "historia de México" se había convertido "en uno de los campos predilectos de la curiosidad y la inteligencia nacionales, y una atracción cada

\footnotetext{
3 Lida, Matesanz y Vázquez, La Casa de España y El Colegio de México. Memoria 1938-2000, p. 203.

${ }^{4}$ Ronan, “¿Qué significa gringo?” (32), 1959, pp. 549-554; VÁzQuez, "Historia Mexicana en el banquillo", pp. 16-18; Miño Grijalva, "Historia Mexicana. Historiografía y conocimiento” (161), 1991, pp. 25-47, esp. pp. 43-46.

${ }_{5}^{5}$ Muro, Historia Mexicana. Índice, pp. iii-v.
} 
vez más general y manifiesta de los estudiosos extranjeros, sobre todo de los norteamericanos". ${ }^{6}$ En el momento en que Cosío Villegas escribió aquel texto, ya El Colegio de México recibía financiamiento de la Fundación Ford. Aun así, buena parte de los recursos que se destinaron a sostener la revista en sus primeros años provino de empresas nacionales: Nacional Financiera, Banco de México, Compañía Fundidora de Fierro y Acero de Monterrey, Banco Agrícola y Ganadero de Toluca...7

La visión histórica de América Latina en la revista estuvo marcada por la presencia de aquel latinoamericanismo universitario de Estados Unidos, pero también por una disputa con la historiografía soviética que todavía no ha sido suficientemente dilucidada. Tras el triunfo de la revolución cubana en 1959 y su radicalización marxista-leninista a partir de 1961, los historiadores soviéticos pusieron a circular una narrativa sobre el siglo XX latinoamericano que reproducía el tópico del "carácter burgués” de la revolución mexicana. Historia Mexicana se convirtió en una plataforma de debate de la historiografía soviética desde que en el número 40, de la primavera de 1961, Lucila Flamand reseñó severamente el libro colectivo de B. T. Rudenko, N. M Lavrov y M. S. Alperóvich, La Revolución Mexicana. Cuatro estudios soviéticos (1960). ${ }^{8}$

Como bien observaba Flamand, siguiendo a Juan Ortega y Medina, la interpretación soviética de la revolución mexicana, en el arranque de la Guerra Fría, reaccionaba contra la lectura positiva del agrarismo, el indigenismo y el nacionalismo cardenistas y postcardenistas en la historiografía estadounidense (Frank Tannenbaum, Robert E. Quirk, E. David Cronon, Charles Cumberland, Stanley R. Ross...). ${ }^{9}$ Fue justamente Ross quien, en otro número de Historia Mexicana, perfiló aquel corpus

${ }^{6}$ Muro, Historia Mexicana. Indice, p. iv.

7 Muro, Historia Mexicana. Índice; Cosío Villegas, Memorias, p. 253.

8 Flamand, “La Revolución Mexicana desde Rusia” (40), 1961, pp. 669-672.

9 Ortega y Medina, Historiografía soviética iberoamericanista, pp. 31-34. 
historiográfico y destacó la importancia de la Hispanic American Historical Review en su difusión. ${ }^{10}$ La historiografía soviética de la Guerra Fría, desde las páginas de la revista Voprosy Istorii, reaccionó contra aquellos análisis insistiendo en las limitaciones de una revolución en que la clase obrera nunca llegó a tomar el poder, dada la escasa industrialización del país.

El debate, que tenía como trasfondo la pugna entre dos modelos de revolución, el mexicano y el cubano, como advirtió muy temprano Daniel Cosío Villegas, tuvo grandes implicaciones para las ciencias sociales latinoamericanas. ${ }^{11}$ En el primer número de Historia Mexicana de 1963, ya consumada la integración de Cuba al bloque soviético, el profesor de la Universidad de Arizona, J. Gregory Oswald, hizo un nuevo cuestionamiento integral del latinoamericanismo soviético, partiendo, otra vez, de Cosío Villegas y Ortega y Medina. ${ }^{12}$ Aunque el foco de atención de Oswald también era la revolución mexicana, su crítica suponía una interpelación de la estrategia integral del Instituto Latinoamericano de la Academia de Ciencias de la URSS, cuyo director a principios de los sesenta, S. S. Mikhailov, sostenía que, en la Guerra Fría, el imperialismo manipulaba a las izquierdas no comunistas latinoamericanas -especialmente, el cardenismo, el varguismo y el peronismo- para retrasar el avance de la causa socialista.

Oswald ya había sido refutado por el historiador soviético I. R. Lavretskii en Voprosy Istorii en 1961 y M. S. Alperóvich había defendido, en la misma revista moscovita, a la historiografía soviética de las críticas publicadas en Hispanic American Historical Review. El nuevo ensayo de Oswald en Historia Mexicana fue respondido por L. I. Slezkin en la revista Novaia Noveishaia Istoriia, en un artículo titulado "Ataque infundado a la historiografía latinoamericana soviética”. Oswald, por su parte,

10 Ross, “México independiente” (42), 1961, pp. 299-313, esp. pp. 305-308.

11 Cosío Villegas, Change in Latin America, pp. 42-54.

12 Oswald, “La Revolución Mexicana en la historiografía soviética” (47), 1963, pp. 340-357. 
respondió a Slezkin en el número 56, de la primavera de 1965. El tema en disputa era México en la historiografía soviética, pero Oswald reconstruyó las líneas generales de la representación de América Latina en las ciencias sociales de la URSS. Con citas de Ponomariov, Mikhailov, Alperóvich, Lavrov y otros historiadores, el profesor de la Universidad de Arizona sostenía que tras la revolución cubana la historiografía soviética había rearticulado algunos clichés sobre la historia latinoamericana del siglo xx (la revolución mexicana había concluido en 1917, México estaba sojuzgado por el imperialismo yanqui, la experiencia cubana era el desenlace de todas las luchas populares latinoamericanas desde la independencia, sólo desde el socialismo era posible realizar los ideales auténticamente revolucionarios...) que era preciso rebatir. ${ }^{13}$

En la primera mitad de los años sesenta, Historia Mexicana se vio involucrada en aquel choque historiográfico de la Guerra Fría. Pero, pese a la sólida presencia de historiadores de Estados Unidos, el tema latinoamericano en la revista fue tratado con matices y modulaciones propios, que no reproducían exactamente las mismas perspectivas de Hispanic American Historical Review y otras publicaciones estadounidenses. Una polémica historiográfica más, en la misma tónica de la Guerra Fría, dejó entrever el latinoamericanismo que se abría paso en la publicación. En el número 62, del otoño de 1966, el diplomático argentino, exiliado en México, Iso Brante Schweide, amigo de Alfredo Palacios, Manuel Ugarte, Mario Bravo y otros socialistas desde los años veinte, $\mathrm{y}$ muy cercano al Instituto Panamericano de Geografía e Historia, criticó tres libros sobre Iberoamérica publicados en Alemania. ${ }^{14}$

Dos de aquellos libros, Alemania, Díaz y la Revolución Mexicana (1964), de Friedrich Katz, y A la sombra de la Santa

13 Oswald, "México en la historiografía soviética” (56), 1965, pp. 691-706, esp. pp. 696-698.

14 Brante Schweide, “Tres libros alemanes con temas americanos y españoles” (62), 1966, pp. 300-306. 
Alianza. Alemania y Latinoamérica (1964), de Manfred Kossok, se habían publicado en la RDA, y el otro, Herencia española y Revolución (1957), de Joachim Fernández, en Westfalia, del lado de la RFA. Brante Schweide, curiosamente, era tan crítico del conservadurismo de Fernández, por "su doliente añoranza de la España de los Habsburgo", como del, a su juicio, afectado antiimperialismo de Katz, quien no "comprendía el hondo significado humanista de la Revolución Mexicana” e idealizaba la "sincera amistad de la Alemania oriental, la Unión Soviética y los países socialistas con México y América Latina”. ${ }^{15}$ En el distanciamiento de Brante Schweide de la historiografía alemana oriental sobre América Latina reaparecía el diferendo con una visión entusiasta de la revolución cubana que relegaba a la mexicana a mero antecedente burgués del proyecto socialista del Caribe.

Aquel diferendo, que Stanley R. Ross enfrentó en su ensayo "Mexico: The Preferred Revolution" (1964), no era tal en la obra de Katz. En su respuesta a Brante Schweide, el autor de La guerra secreta en México (1981) dejaría claro que no compartía la esquemática valoración de la revolución mexicana que se reproducía en la historiografía soviética y destacaba su interlocución con historiadores mexicanos como Daniel Cosío Villegas, Luis González y González, Moisés González Navarro, Fernando Rosenzweig y Berta Ulloa. ${ }^{16}$ Ninguno de aquellos historiadores mexicanos jerarquizaba ideológicamente las dos grandes revoluciones latinoamericanas del siglo $\mathrm{xx}$ a favor de la socialista cubana y ninguno de ellos compartía la percepción de la revolución mexicana u otras experiencias de la izquierda latinoamericana no comunista, de la primera mitad del siglo xx, como proyectos burgueses.

${ }_{15}$ Brante Schweide, "Tres libros alemanes con temas americanos y españoles" (62), 1966, pp. 301-302 y 304.

16 Katz, "Friedrich Katz responde a Iso Brante Schweide" (64), 1967, pp. 621-624, esp. p. 624. 
El latinoamericanismo de Historia Mexicana se alojó en aquel flanco y avanzó, entre los años cincuenta y sesenta, a través de dos rutas historiográficas fundamentales: la historia de las ideas y la historia de las relaciones internacionales. Los principales libros de Leopoldo Zea sobre el pensamiento hispanoamericano, en aquellas décadas -Dos etapas del pensamiento en Hispanoamérica (1949), América como conciencia (1953), América en la bistoria (1957), El pensamiento latinoamericano (1965)-, fueron reseñados y comentados, elogiosamente, por María del Carmen Velázquez, María Elena Rodríguez de Magis y Charles Hale. En una de aquellas notas Velázquez resumía que la visión de la historia de las ideas continentales de Zea proponía un distanciamiento del "panamericanismo" y, a la vez, una postulación de Hispanoamérica como lugar de enunciación de la filosofía occidental, pero también como comunidad lo suficientemente diversa como para producir recepciones nacionales distintas de corrientes europeas como el liberalismo y el positivismo. ${ }^{17}$

Autora, ella misma, del importante volumen de historia internacional, Hispanoamérica en el siglo XIX (1964), reseñado por Francisco Xavier Tapia, María del Carmen Velázquez mantuvo un interés permanente en la historiografía latinoamericanista, con especial énfasis en las relaciones entre Estados Unidos y las naciones al sur del río Bravo. ${ }^{18}$ A la vez que en Zea o en Velázquez se alternaban enfoques "latinoamericanos" o "hispanoamericanos" de la historia continental, en la obra de Silvio Zavala se delineaba una perspectiva “americanista”, compartida por historiadores como Edmundo O’Gorman o Daniel Cosío Villegas. El ensayo de Zavala, "Rivalidades imperiales en el Nuevo Mundo” (1963), transmitía una visión integrada de la historia

17 Velázquez, “Pensamiento hispanoamericano” (2), 1951, pp. 323-325, esp. p. 325.

18 Velázquez, “La política de Estados Unidos en América Latina” (40), 1961, pp. 667-669; TAPIA, "María del Carmen Velázquez, Hispanoamérica en el siglo XIX” (61), 1966, pp. 85-86. 
continental, en la que hispanoamericanos, lusoamericanos, angloamericanos y, en menor medida, francoamericanos, experimentaban un devenir compartido y, a la vez, diferenciado. ${ }^{19}$ Esa perspectiva era muy similar a la sostenida por el propio Zavala en la Revista de Historia de América, editada por el Instituto Panamericano de Geografía e Historia desde fines de los años treinta, y por sus propios discípulos, reunidos en un homenaje que le rindió Historia Mexicana en el primer número de 1955. ${ }^{20}$

\section{EL LATINOAMERICANISMO ESTRUCTURALISTA}

La coexistencia de enfoques latinoamericanistas y hemisféricos en Historia Mexicana se mantuvo en el tránsito a la Guerra Fría tardía durante los años setenta. Todavía en el índice temático realizado por Luis Muro en 1971 no aparecía América Latina o Latinoamérica o Hispanoamérica, sino simplemente "América" ${ }^{21}$ Sin embargo, desde ese mismo año, el giro historiográfico favorable al marxismo y el estructuralismo, la historia económica y la historia social, comenzó a traducirse en un latinoamericanismo que insertaba a México en perspectivas continentales, cercanas a las posiciones desarrollistas y, en menor medida, socialistas, que predominaban en círculos teóricos como los de la CEPAL y la teoría de la dependencia. La presencia de Enrique Florescano y Alejandra Moreno Toscano, ambos formados en París con apoyo de Zavala, en el núcleo editorial de la publicación, fue decisiva en aquel giro.

Los profesores de Princeton, Stanley y Barbara Stein, habían publicado en 1970 un ensayo clásico, The Colonial Heritage of Latin America, reseñado en la revista por Miguel Marín Bosch. $\mathrm{Al}$ año siguiente, en el número 82, dedicado a conmemorar el

19 Zavala, "Rivalidades imperiales” (47), 1963, pp. 325-339.

20 "Estudios históricos americanos", pp. 407-422; PANI, "Silvio Zavala y la historia de América” (155), 2018, pp. 177-189.

21 Muro, Historia Mexicana. Índice, p. 101. 
vigésimo aniversario de la publicación, entonces bajo la dirección de Enrique Florescano, se tradujo un enjundioso ensayo de Stanley Stein y Shane Hunt sobre la historia económica del continente. Bajo el prisma de la teoría dependentista y el socialismo cubano, estos historiadores llamaban a ir más allá de la sustitución de importaciones y la dilatación del mercado interno, en una estrategia de desarrollo de las economías latinoamericanas que superara la fase "neocolonial". ${ }^{22}$

La centralidad que adquirió la historia económica se reflejó en una mayor presencia de visiones de conjunto sobre la experiencia del continente en la larga duración. Héctor Aguilar Camín reseñó el volumen La historia económica en América Latina (1972), editado por la SEP, que reunió estudios de Tulio Halperín Donghi sobre Argentina, Francisco Iglesias sobre Brasil, Thomas Mathews sobre el Caribe, Juan Maiguashca sobre Ecuador, Juan Oddone sobre Uruguay y Enrique Florescano sobre México. Alejandra Moreno Toscano comentó el clásico estudio de Marcello Carmagnani, L' America Latina dal 500 a oggi (1975). Jesús Monjarás-Ruiz se ocupó de la historia socioeconómica de Centroamérica de Murdo J. MacLeod en Spanish Central America (1973). Andrés Lira examinó otro clásico, Studies in Colonial History of Spanish America (1975) del historiador chileno Mario Góngora, y Jan Bazant atendió los ensayos de Stanley Stein, Roberto Cortés Conde, Kenneth Duncan, Ian Ruthlegde, Robert G. Keith y François Chevalier.

El recorrido por aquellas reseñas en Historia Mexicana, durante los años setenta, da cuenta de la presencia de América Latina en la revista y, a la vez, del rico debate teórico sobre las estructuras de la economía latinoamericana en aquella década. Mientras Stein hablaba de un sistema "neocolonial", Carmagnani prefería el adjetivo "feudal”, que, en buena medida, rechazaba

22 Stein y Hunt, “La historia económica en América Latina” (82), 1971, pp. 328-371. 
Góngora en sus estudios. ${ }^{23}$ Aquella discusión, como sugería Bazant en su nota sobre L'Amérique Latine de l'indépendence à nos jours (1977) de François Chevalier, no era un asunto del pasado, ya que fenómenos como la revolución mexicana, el peronismo, el varguismo y, sobre todo, el cardenismo, no podían entenderse sin la historia previa del latifundismo latinoamericano. ${ }^{24}$

A diferencia del momento de auge de la historia económica, la difusión que alcanzó la historia social y cultural, de las mentalidades y los imaginarios, de la vida privada y cotidiana, en los años ochenta, en buena medida por influencia de la nouvelle histoire francesa, no se tradujo en un reforzamiento del latinoamericanismo de Historia Mexicana. Libros como The Frontier in Latin American History (1978) de Alistair Hennessy o Notable Family Networks in Latin America (1984) de Diana Balmori, Stuart F. Voss y Miles Wertman, interesaron a María del Carmen Velázquez y a María de los Ángeles Romero Frizzi. América Latina perdió presencia en la revista en los ochenta y comenzó a recuperarla a fines de la década, cuando se instala el debate sobre el V Centenario de la llegada de Cristóbal Colón a estas tierras.

La decadencia de los socialismos reales en los años ochenta, la caída del Muro de Berlín en 1989 y la desintegración de la URSS entre 1991 y 1992 tuvieron un impacto poco reconocido y estudiado en la producción intelectual latinoamericana. Aquellos años, que coincidieron con las transiciones a la democracia desde las últimas dictaduras militares de la Guerra Fría, fueron fértiles en debates sobre la crisis del marxismo, el fenómeno del

${ }^{23}$ Moreno Toscano, "Sobre Marcello Carmagnani, L'America Latina dal 500 a oggi-Nascita, espansione e crisi di un sistema fendale" (99), 1976, pp. 498500; Lira GonzÁlez, "Sobre Mario Góngora, Studies in colonial history of Spanish America” (95), 1977, pp. 149-153; GóNGORA, Estudios sobre la bistoria colonial de Hispanoamérica, pp. 63-65 y 116.

${ }^{24}$ Bazant, "Sobre François Chevalier, L'Amérique Latine de l'indépendance à nos jours" (111), 1979, pp. 482-484. 
nacionalismo y las identidades culturales. Tres libros aparecidos en 1983 y con frecuentes reediciones en años siguientes, Imagined Communities (1983) de Benedict Anderson, The Invention of Tradition (1983) de Eric Hobsbawm y Nations and Nationalism (1983) de Ernest Gellner, dejaron una impronta discernible en la historiografía latinoamericana.

El cambio global que se produjo entre 1985 y 1990 fue propicio para la renovación de la historia política, cultural e intelectual de la región. Junto con una inmersión en los estudios sobre las identidades y los nacionalismos, comenzó a producirse una rearticulación de la perspectiva iberoamericana que recibió impulso diplomático desde España, Portugal y diversos países del continente. La creación del mecanismo de las Cumbres Iberoamericanas, en 1991 en Guadalajara, y de la institución de la Secretaría General Iberoamericana, además de la conmemoración de los quinientos años del arribo de Colón a América en casi todas las capitales latinoamericanas, crearon una plataforma favorable al enfoque iberoamericano.

Aquel giro al iberoamericanismo, estrechamente ligado al fin de la Guerra Fría, se manifestó de múltiples formas en Historia Mexicana. Señal inequívoca de la nueva época fue la aparición, en el número cuarto de 1995, del ensayo-autocrítica de M. S. Alperóvich, historiador soviético fuertemente involucrado en los combates ideológicos de los años cincuenta, sesenta y setenta. Alperóvich reconocía, ahora, que historiadores críticos de la escuela soviética como Gregory Oswald y Juan A. Ortega y Medina tenían razón al cuestionar el supuesto “carácter democrático-burgués” de la revolución mexicana y las izquierdas populistas y adjudicaba el error a que "aquellos trabajos fueron escritos por historiadores formados bajo la influencia de la ideología comunista”. ${ }^{25}$ Historiadores, agregaba Alperóvich,

25 Alperóvich, "La revolución mexicana en la interpretación soviética del periodo de la 'Guerra Fría”' (176), 1995, pp. 678-686, esp. p. 678. 
"egresados de escuelas y universidades soviéticas, en las que se les persuadía con insistencia, la idea de la infalibilidad, la impecabilidad y la universalidad de la llamada metodología marxista-leninista". ${ }^{26}$

Recordaba también Alperóvich las campañas de censura o descalificación emprendidas por jerarcas del Partido Comunista y la Academia de Ciencias de la URSS, como el lituano I. R. Grigulevich (a.k.a. I. R. Lavrteskii), que había sido agente de la NKVD en México a fines de los treinta y organizador del atentado de David Alfaro Siqueiros contra León Trotski. Según Alperóvich, Lavretskii (Grigulevicth) no sólo había sido el artífice de los ataques a la visión de la revolución mexicana que trasmitían publicaciones como The Hispanic American Historical Review o Historia Mexicana, sino que había confrontado a historiadores soviéticos revisionistas, como L. I. Zubok, quien en su temprano estudio La política imperialista en el área del Caribe (1948) valoraba positivamente la experiencia revolucionaria mexicana. ${ }^{27}$

\section{AUGE Y CRISIS DEL IBEROAMERICANISMO}

El quinto centenario de Colón en América comenzó a debatirse en Historia Mexicana mucho antes de octubre de 1992. La formación de comisiones nacionales que organizarían las celebraciones, en cada país de la región, comenzó desde mediados de los ochenta, bajo el impulso del primer gobierno de Felipe González en España. En 1984 tuvo lugar una primera reunión de miembros de aquellas comisiones en Santo Domingo, República Dominicana, en la que participó Miguel León Portilla a nombre de México. Allí el profesor de la UNAM proponía no definir los actos por el V Centenario como "celebración"

\footnotetext{
26 Alperóvich, "La revolución mexicana en la interpretación soviética del periodo de la “Guerra Fría”" (176), 1995, pp. 678-686.

27 Alperóvich, "La revolución mexicana en la interpretación soviética del periodo de la 'Guerra Fría”" (176), 1995, pp. 679 y 684-686.
} 
sino como conmemoración, y no llamar lo que sucedió el 12 de octubre de 1492 "descubrimiento" sino “encuentro de dos mundos que habían permanecido totalmente ajenos el uno al otro hasta fines del siglo $\mathrm{XV}$ ". ${ }^{28}$

El debate sobre los 500 años de la llegada de Colón quedó abierto desde entonces en México, como se lee en publicaciones como La Jornada Semanal y Cuadernos Americanos. Edmundo O’Gorman respondió a León Portilla con la doble negación de "ni descubrimiento ni encuentro", ya que, según sus propias investigaciones, en los viajes de Colón, América había sido inventada como parte oriental extrema de las Indias y, por la misma razón, los europeos no habían encontrado aquí algo equivalente a un "Nuevo Mundo". ${ }^{29}$ En Historia Mexicana, una de las primeras incursiones en el tema fue del historiador estadounidense Lewis Hanke, especializado en los gobiernos virreinales americanos.

Hanke no dudaba de que había que "celebrar" y "conmemorar" el V Centenario. El "cariño" que se le tenía a hitos como el 12 de octubre de 1492, argüía, estaba fundado en "lo que Richard M. Morse llamaba la forma casuística tomística ibérica de ver la vida". ${ }^{30}$ Hanke sostenía que la mejor manera de celebrar era la investigación a fondo del fenómeno de la conquista y evangelización de las Indias, rehuyendo los tópicos ideológicos, e involucrando a investigadores más allá del mundo hispánico. Profesor de las universidades de Harvard, Columbia y Austin, Hanke ponía como ejemplos sus propios estudios sobre Bartolomé de Las Casas, los del también estadounidense Samuel Eliot Morison sobre Cristóbal Colón y los del hispanista sueco

28 Gómez Robledo, “Descubrimiento o encuentro” (146), 1987, pp. 285-297, esp. p. 285.

29 Gómez Robledo, “Descubrimiento o encuentro" (146), 1987, pp. 286-287. 30 Hanke, “¿Cómo deben conmemorarse los quinientos años del descubrimiento de América?” (145), 1987, pp. 101-113, esp. p. 101. 
Magnus Mörner sobre el mestizaje como uno de los elementos constitutivos de la cultura latinoamericana. ${ }^{31}$

El veterano intelectual católico mexicano Antonio Gómez Robledo también intervino en aquel debate, defendiendo, a partir de las viejas tesis panhispanistas de Carlos Pereyra, la necesidad de celebrar el descubrimiento de América con todas sus letras. ${ }^{32}$ Se remontaba Gómez Robledo a la querella neotomista sobre el derecho de gentes, en el siglo xvI, por medio de glosas de teólogos como el escocés John Maior o los españoles Juan López de Palacios Rubios, Ginés de Sepúlveda y Francisco de Vitoria, con el fin de reafirmar el tópico colonial de que la civilización hispánica era "superior" a la mexica. ${ }^{33}$ Pero Gómez Robledo era lo suficientemente diplomático como para registrar otras posiciones como las de los filósofos Leopoldo Zea y Enrique Dussel, quienes desde perspectivas diversas también rechazaban el concepto de "encuentro de dos mundos", debido a que el mundo conquistador, según el primero, "encubría” al conquistado o "intentaba constituirlo en ente explotable", al decir del segundo. ${ }^{34}$

En los años noventa, conforme avanzaba el nuevo iberoamericanismo, repuntaba el perfil de la región en Historia Mexicana. Varios ejercicios de historia comparada colonial, como los de las despoblaciones mexicana y peruana en los siglos xvi y xviI de Carlos Sempat Assadourian, las conexiones sevillanas de esos mismos virreinatos de Teodoro Hampe, la desregulación comercial en Nueva España y Cuba durante el reinado de Carlos III de Allan J. Kuethe o el repaso de la historiografía argentina sobre los siglos XvII y xviII de Enrique Tandeter se publicaron a principios de aquella década. Clara Lida, que había asumido

31 Hanke, “¿Cómo deben conmemorarse los quinientos años del descubrimiento de América?” (145), 1987, p. 102.

32 Gómez Robledo, “Descubrimiento o encuentro” (146), 1987, p. 287.

33 Gómez Robledo, “Descubrimiento o encuentro” (146), 1987, p. 294.

34 Gómez Robledo, “Descubrimiento o encuentro” (146), 1987, pp. 297-298. 
la dirección de la revista en enero de 1989, señalaba en la introducción al número 161, en el verano de 1991, que a 40 años de la fundación de Historia Mexicana, una de las "novedades" era la "apertura a la historia comparada que situaba a México en su hábitat histórico por antonomasia: Latinoamérica”. ${ }^{35}$

En 1993 pudo constatarse aquella apertura hacia América Latina en el número 167, que incluyó ensayos de Jaime E. Rodríguez O. sobre las independencias en la América española, de Roberto Cortés Conde sobre el crecimiento de las economías latinoamericanas entre 1880 y 1930, y de Tulio Halperín Donghi sobre lo que llamaba la "reflexión hispanoamericana" o las diversas formas en que los hispanoamericanos habían debatido su experiencia histórica moderna, desde la Carta de Jamaica de Simón Bolívar hasta los influyentes ensayos de Claudio Véliz y Hernando de Soto en los años ochenta. ${ }^{36}$ El ambicioso recorrido de Halperín Donghi se detenía en los sucesivos momentos de la tradición intelectual hispanoamericana, el republicano (Bolívar, Mier, Bello), el liberal (Sarmiento, Alberdi, Lastarria, Otero), el positivista (Bulnes, Sierra, los hermanos García Calderón), el revolucionario (Haya de la Torre, Mariátegui, Vasconcelos), para desembocar en los dilemas irresueltos por las tesis de la CEPAL, la teoría de la dependencia y el socialismo cubano, que veía plasmados en los ensayos de Véliz y Soto sobre la paradoja del centralismo y la informalidad en América Latina. ${ }^{37}$

En la presentación del número 169, en el verano de 1993, Alicia Hernández Chávez, entonces directora del Centro de Estudios Históricos, apuntaba que desde 1989 El Colegio de México había decidido consolidar la presencia de estudiantes

35 Lida, "Hacia la quinta década” (161), 1991, pp. 8-10, esp. p. 9.

36 Halperín Donghi, "Hispanoamérica en el espejo" (167), 1993, pp. 745787pp. 745-787.

37 Halperín Donghi, "Hispanoamérica en el espejo" (167), 1993, pp. 783-785. 
latinoamericanos en sus programas doctorales. El objetivo era que esos estudiantes profundizaran en el conocimiento "no sólo de la historia de México sino también la de sus países, a partir de la perspectiva comparada". ${ }^{38}$ Aquella premisa se confirmó en el número 180, de la primavera de 1996, coordinado por la historiadora puertorriqueña Laura Náter y el profesor Carlos Marichal, quien, con sus estudios sobre la deuda externa, las inversiones extranjeras, las crisis financieras y las relaciones diplomáticas continentales, se convertiría en uno de los referentes centrales del latinoamericanismo en la historiografía mexicana entre fines del siglo xx y principios del XxI. ${ }^{39}$ Con artículos de la propia Náter, Mabel Rodríguez, Teresa Maya, Marina Zuloaga, José Antonio Serrano y María Cecilia Zuleta, aquel número abordó el reconocimiento de la independencia de América Latina por España, las relaciones comerciales entre México y Estados Unidos en el siglo xIx, la Conferencia Internacional Americana de 1889 y el primer panamericanismo, la política mexicana hacia la independencia de Cuba, la diplomacia española frente a la revolución mexicana, los proyectos de unificación centroamericana y las iniciativas diplomáticas de Alfonso Reyes en Argentina.

Los temas latinoamericanos se afincaron en la revista en los años noventa con estudios de Rafael Sagredo sobre los catecismos cívicos hispanoamericanos a principios del siglo XIX y de Pablo Yankelevich sobre el intelectual argentino Manuel Ugarte y su visión de la revolución mexicana. El debate entre Tulio Halperín Donghi, John Tutino y Florencia E. Mallon sobre las rebeliones campesinas y la construcción nacional en América Latina, aparecido en el primer número de 1997, fue muy revelador de la inscripción de Historia Mexicana en un campo historiográfico que tomaba distancia de la concepción meramente discursiva de

\footnotetext{
38 Hernández Chávez, "Presentación” (169), 1993, pp. 3-5, esp. p. 3.

39 NÁter y Marichal, "Una mirada hacia afuera” (180), 1996, pp. 699-704.
} 
los nacionalismos y que otorgaba gran importancia a las prácticas de resistencia de los sectores populares. ${ }^{40}$

Aunque perdió fuerza en la primera década del siglo xxi, la perspectiva latinoamericana persistió en los estudios de Guillermo Palacios sobre las relaciones entre México y Brasil y en los ensayos sobre la construcción de la identidad nacional en América Latina, Argentina y Cuba de los historiadores españoles Francisco Colom González y Consuelo Naranjo Orovio y la argentina Mónica Quijada, convocados por Tomás Pérez Vejo. ${ }^{41}$ Con estos estudios se produjo, en Historia Mexicana, una vuelta a la línea crítica de los nacionalismos, que tomaba distancia de la historia social defendida por Florencia Mallon, John Tutino y Eric Van Young, entre otros importantes historiadores.

La coyuntura del bicentenario de los procesos de independencia, entre 2008 y 2010, volvió colocar a América Latina en el centro de la publicación, aunque reiterando el enfoque hispánico. El número 229, del verano de 2008, coordinado por José A. Piqueras, atrajo colaboraciones de Antonio Annino, José María Portillo Valdés, Antonio Moliner Prada, Anthony McFarlane, José Carlos Chiaramonte, Luis Miguel Glave y Beatriz Rojas. Si bien algunos de aquellos ensayos estudiaron casos americanos particulares, como los de la Nueva España, el Perú, el Río de la Plata o Cuba, el eje argumentativo del dossier preservaba y, a la vez, matizaba la tesis de la "revolución hispánica", sostenida por François-Xavier Guerra, Jaime E. Rodríguez O. y otros autores. ${ }^{42}$

40 Halperín Donghi, “Campesinado y nación” (183), 1997, pp. 503-529; Tutino, “La negociación de los estados nacionales” (183), 1997, pp. 531-562; Mallon, "En busca de una nueva historiografía latinoamericana” (183), 1997, pp. 563-580.

41 PÉrez Vejo, "La construcción de las naciones como problema historiográfico” (210), 2003, pp. 275-311.

42 Piqueras, "Revolución en ambos hemisferios" (229), 2008, pp. 31-98. 
En el verano de 2010, el número 237, coordinado por Tomás Pérez Vejo, volvió a tratar el tema de las revoluciones hispánicas. Esta vez el prisma elegido fue el de la cultura representacional de los centenarios y las conmemoraciones de las independencias de 1810, en 1910, por lo que el arco territorial incluyó diversos casos nacionales del mundo hispánico: Paraguay, Venezuela, Chile, Argentina, Ecuador, Colombia, México y, por supuesto, España. ${ }^{43}$ Este número, el más abarcador de todos los dedicados a la región, en Historia Mexicana, partió de aquella premisa introducida por Anderson, Gellner, Hobsbawm y otros autores, desde los años ochenta del siglo xx, de que los nacionalismos eran procesos de construcción imaginaria fuertemente endeudados con las liturgias y los ceremoniales de legitimación del Estado. ${ }^{44}$

Tanto el número 229, coordinado por Piqueras, como el 237, coordinado por Pérez Vejo, implicaron un desplazamiento del horizonte iberoamericano trazado en los años noventa hacia nuevas coordenadas interpretativas de lo hispánico. Esto no supuso un abandono de enfoques latinoamericanistas como los que se desprenden de colaboraciones de autores frecuentes, de varias generaciones de El Colegio de México, como Marco Palacios, Francisco Zapata, Pablo Yankelevich, Cecilia Zuleta, Alexandra Pita o Aimer Granados. Además de ser una de las plataformas fundamentales de la producción historiográfica nacional, Historia Mexicana no ha dejado de ser un espacio de diálogo y debate del saber histórico en América Latina y el Caribe.

\section{REFERENCIAS}

Cosío Villegas, Daniel, Change in Latin America. The Mexican and the Cuban Revolutions, Lincoln, The University of Nebraska, 1961.

43 Pérez Vejo, “Los centenarios en Hispanoamérica” (237), 2010, pp. 7-29.

44 Pérez Vejo, “Los centenarios en Hispanoamérica” (237), 2010, pp. 14-15. 
Cosío Villegas, Daniel, “Diez años de camino”, en Muro, 1961, pp. iii-v.

Cosío Villegas, Daniel, Memorias, México, Joaquín Mortiz, 1976.

Góngora, Mario, Estudios sobre la historia colonial de Hispanoamérica, Santiago de Chile, Editorial Universitaria, 1998.

Lida, Clara E., José Antonio Matesanz y Josefina Zoraida Vázquez, La Casa de España y El Colegio de México. Memoria. 1938-2000, México, El Colegio de México, 2000.

Muro, Luis (ed.), Historia Mexicana. Índice de sus primeros diez años, México, El Colegio de México, 1961.

Ortega y Medina, Juan A., Historiografía soviética iberoamericanista. 19451960. Obras. 5, México, Universidad Nacional Autónoma de México, 2018. 